\title{
Formation of star clusters: Models and simulations
}

\author{
Philip C. Myers \\ Harvard-Smithsonian Center for Astrophysics \\ 60 Garden Street, Cambridge, MA 02138 USA \\ email: pmyers@cfa.harvard.edu
}

\begin{abstract}
Recent progress is reviewed in describing the physical processes of star cluster formation, from the viewpoint of theoretical models and numerical simulations. In many studies, supersonic turbulent flows structure the gas into a network of clumps, filaments, and cores. Selfgravity drives star formation, moderated by turbulence, magnetic fields, and stellar feedback. Recent models and simulations describe the core mass function, the protostar mass function, and the star-formation rate. Several simulations indicate similar protostar birthrates, a mass advantage for firstborn protostars, and accretion rates that are superthermal and nearly constant. Recently observed core-filament systems with sonic line widths and parsec lengths present challenges for turbulent models.
\end{abstract}

Keywords. Molecular clouds, star formation, star clusters

\section{Introduction}

Embedded clusters are parsec-scale concentrations of dense gas and very young stars. On time scales of a few Myr, their dense cores form protostars, their protostars gain mass, and the circumstellar disks around their pre-main sequence stars begin to form planets. These regions provide the best laboratories to observe the processes of star formation. Recent observational reviews include Longmore et al. (2014), Luhman (2012), Portegies Zwart et al. (2010), Allen et al. (2007), and Zinnecker \& Yorke (2007).

The processes of star formation in clusters have also been the subject of an increasing number of theoretical and numerical studies. Large-scale numerical simulations have greatly extended the reach of analytical models. Recent reviews of cluster theories and simulations address the initial mass function (Offner et al. 2014), formation of massive stars (Tan et al. 2014), the role of stellar feedback (Krumholz et al. 2014), and theoretical aspects of cluster formation (McKee \& Ostriker 2007).

This review discusses recent developments that shed light on the key questions:

(1) Is cluster formation slow or fast? Can cluster formation be described by quasiequilibrium models where turbulence and magnetic forces oppose gravity over many initial free-fall times (Tan et al. 2006; Huff \& Stahler 2007)? Or is a more dynamic picture needed, where supersonic turbulent flows and stellar feedback act over only a few free-fall times (Ballesteros-Paredes et al. 1999; Elmegreen 2000; Bonnell et al. 2001)?

(2) Do massive stars in clusters form first or last? Do the most massive stars start accreting before lower-mass stars, in the deepest parts of the potential well, as in some simulations (Bonnell et al. 1997; Wang et al. 2010), or do they form later, when their winds and feedback disperse dense gas and shut off further star formation (Herbig 1962)?

(3) Do cluster protostars gain mass at a rate expected from models of "collapse" or "accretion"? The standard model of isolated low-mass star formation (Shu et al. 1987) relies on the constant accretion rate due to collapse of a singular isothermal sphere 


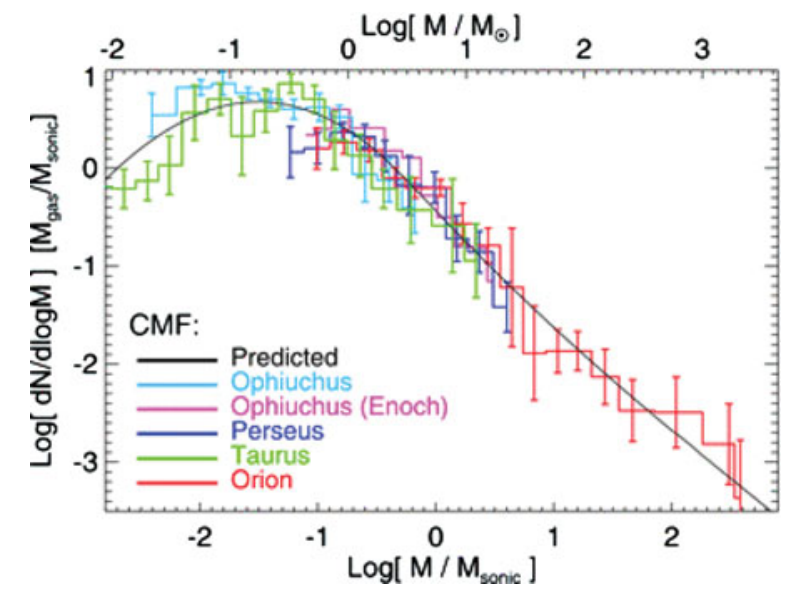

Figure 1. Predicted core mass function, based on a log-normal distribution of density fluctuations in a turbulent medium, which at each spatial scale are dense enough to be gravitationally bound (Hopkins 2012). The distribution agrees well with observed estimates of the core mass function. (A color version of this figure is available online.)

(SIS - Shu 1977), while more massive stars are expected to gain mass more rapidly, by gravitational accretion, whose rate increases with protostar mass (Zinnecker 1982; Bonnell et al. 1997).

\section{Analytic models}

Here, three analytic models are described, which match theoretical predictions to observational data on young clusters and regions of star formation.

Accelerating star formation. The ages of pre-main sequence (PMS) stars in Orion, based on photometric and spectroscopic observations, and on models of PMS evolution, indicate that most PMS stars were born in the last 1-2 Myr, but their age distribution has a tail extending to ages of $\sim 10 \mathrm{Myr}$ (Palla \& Stahler 2000). This distribution is well fit by a quasi-equilibrium model of spherical clump contraction against the slow dissipation of turbulent support due to colliding shocks (Tan et al. 2006; Huff \& Stahler 2007). Then the protostar births "accelerate" as the clump collapses.

Core mass function (CMF). In these models, the CMF is the mass distribution of gravitationally bound density fluctuations on all spatial scales, where a fluctuation is bound if its mass equals the Jeans mass corresponding to the thermal and turbulent velocity dispersion. These models require proper counting of small bound condensations that lie inside larger bound condensations, the "cloud-in-cloud problem." Fig. 1 shows a good match of observed and predicted CMFs. However, it is based on a relatively large turbulent Mach number, $M=30$ (Hopkins 2012).

Star-formation rate. An important consequence of the core mass function is the rate at which cores form stars. This regional property can be expressed as the gain rate of the mass surface density of stars $(d / d t) \sum_{\text {stars }}$ as a function of the mass surface density of gas $\sum_{\text {gas }}$. The empirical Kennicutt-Schmidt $(\mathrm{KS})$ relation, $(d / d t) \sum_{\text {stars }} \propto \sum_{\text {gas }}^{p}$, where $p \sim 1.4$, applies over a wide range of galactic and extragalactic regions (Schmidt 1959; Kennicutt 1998). The CMF models of Hennebelle \& Chabrier (2008) predict KS relations for Milky Way clouds of various sizes and for extragalactic clouds (Chabrier et al. 2014). These models match observed KS data with significant scatter at low surface density. 


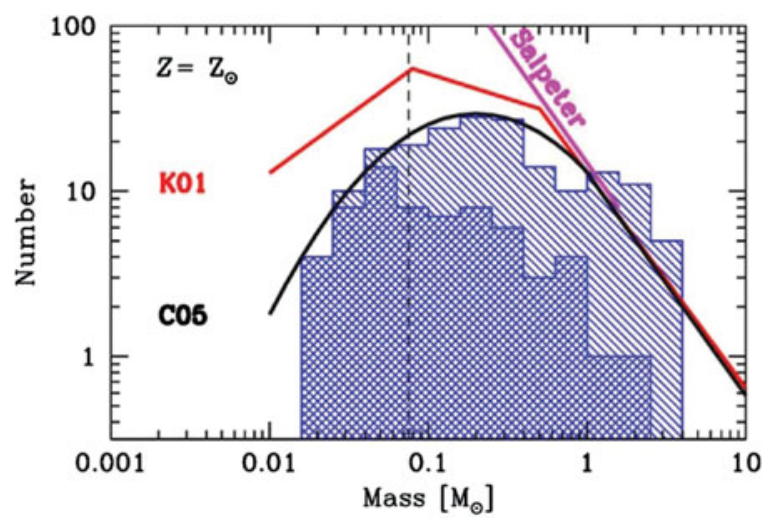

Figure 2. Mass functions of stars that have stopped accreting (heavy shading) and that are still accreting at the end of the simulation (light shading). The lines indicate the IMF distributions of Salpeter (1955), Kroupa (2001), and Chabrier (2005). Adapted from Bate (2014).

\section{Numerical simulations}

Analytic models give physical insight into broad properties of cluster formation but cannot account for the complex structure and evolution of embedded clusters. In contrast, numerical simulations of forming clusters have improved in resolution, computational power, and run time. Now one can vary the initial gas configuration, the strength and geometry of the associated magnetic field, and properties of stellar feedback (e.g., Federrath 2015; Körtgen \& Banerjee 2015; Bate 2014; Myers et al. 2014).

Methods. Many simulations compare results with stellar and gas properties, including the distributions of stellar masses and multiplicity, and the rate of star formation. The typical protocluster has initial properties of several hundred $M_{\odot}$ and several hundred Jeans masses in a 1-pc clump, with turbulent hydrodynamic and Alfvénic Mach numbers $\sim 10$. The turbulent motions stir the initial gas into a clumpy, filamentary network.

The turbulent motions may be generated by a large-scale "turbulence driver" that causes the gas to have velocity dispersion $\sigma$ increasing with size scale $R$, as in the Larson relation $\sigma \sim R^{q}$, where $q \approx \frac{1}{2}$ (Federrath 2015). Alternately, colliding supersonic flows can form a turbulent, filamentary layer (Vázquez-Semadeni et al. 2006).

The evolving clump is followed for about a free-fall time corresponding to its initial mean density. Its densest gas collapses into sink cells, which are taken as protostars if the simulation has sufficient resolution. The evolution is terminated when the mass in protostars has reached a fraction $\sim 10 \%$ of the total mass, as in observed clusters.

Matching the IMF. Simulations of turbulent fragmentation, using a smooth particle hydrodynamics code with resolution $\sim 20$ AU, make enough protostars to compare their mass distribution with the IMF, as the metallicity of the gas is varied (Bate 2014). These simulations include radiative heating from their recently formed protostars. This heating inhibits the formation of low-mass stars and brown dwarfs, and thereby provides mass distributions in relatively good accord with the IMF (Bate 2012).

In these studies, it was found that the protostar mass functions do not vary significantly as the metallicity of the gas changes from 0.01 to 3 times the solar value. Fig. 2 shows the protostar mass distribution for solar metallicity. This figure also shows that the mass function arising from the simulation agrees approximately with estimates of the field star IMF, within statistical uncertainty.

Matching observed star-formation rates. To be realistic, such simulations must also match the rate of star formation estimated from observations of young clusters. The 
earliest hydrodynamic simulations made protostars at too great a rate, because their initial clumps were too dense and because they did not include enough physical processes that oppose gravity and disperse star-forming gas. Recent simulations using the FLASH adaptive mesh refinement code found that the rate of star formation is reduced by a factor 2-3 from that due to gravity alone, as the effects of turbulent stirring, magnetic fields and outflow feedback are sequentially included (Federrath 2015). This rate reduction was realized by running each simulation until it formed $20 \%$ of the initial mass into sink cells. In combination, these effects increase the star-forming life of a young cluster by an order of magnitude, bringing the mean star-formation rate to within a few times the typical observational estimate of a few $M_{\odot} \mathrm{pc}^{-2} \mathrm{Myr}^{-1}$. These results extend and confirm the pioneering study of Wang et al. (2010).

\section{Protostar accretion histories}

As numerical simulations of cluster formation have become more realistic, it has become possible to study the mass accretion histories $m(t)$ of their sink cells, which are identified as young stellar objects (YSOs). Accretion histories are useful to understand the protostar birth rate, the gain rate of protostar mass, and the nature of accretion stopping. These processes are essential parts of how protostars gain their mass, but observations cannot sample protostar masses over a sufficiently long time span.

A recent study compares the accretion histories of YSOs in four hydrodynamic simulations carried out by groups using different codes and initial conditions (Myers et al. 2016). The simulations, by Bate (2012), Krumholz et al. (2012), Girichidis et al. (2011), and Wang et al. (2010), have initial clumps with 500-1200 $M_{\odot} \log$ mean density $\left(\right.$ in $^{\mathrm{cm}^{-3}}$ ) of 4-6 and turbulent Mach number 3-15. Each simulation produces 100-200 YSOs over $0.5-1.3$ initial free-fall times.

In each simulation, the accretion histories allow a clear distinction between two kinds of YSO. At the end time of the simulation, some YSOs have rising $m(t)$ while others have flat $m(t)$. A rising $m(t)$ is interpreted as due to a protostar (PS) that is still accreting, while a flat $m(t)$ is interpreted as due to a pre-main sequence star (PMS), which has essentially stopped accreting.

Birth history. Each simulation shows a continuous history of protostar births, at a mean rate $\sim 100$ births per free-fall time. Some birth histories have a nearly constant rate, while others show a gradual acceleration or a "burst" as a new subcluster is born. Some simulations resemble very young clusters, where PS outnumber PMS stars, while others resemble more evolved clusters, where PMS stars outnumber PS.

Early-birth mass advantage. Most of the simulations also show a tendency for earlyborn stars to become relatively massive. This early-birth mass advantage appears both as an excess of early-born stars with high final mass and as a deficit of early-born stars with low final mass, as first noted by Wang et al. (2010) and by Bate (2012).

Mass accretion rates. In these simulations, the form of the average PS accretion history, $\langle m(t)\rangle$, is remarkably linear. Individual protostar accretion histories $m(t)$ typically fluctuate about a rising trend. But when $\sim 10$ histories having similar start times are averaged together, the fluctuations are reduced, and the resulting average history can be fit with a simple linear function, as shown in Fig. 3.

The constant superthermal mass accretion rates in Fig. 3 differ from those expected in well-known models. The rates exceed that of the collapsing SIS by more than an order of magnitude. Such an increase could arise from collapse of a configuration whose initial density exceeds that of the SIS by a large factor (Shu 1977), but such an initial state is highly unlikely. The factor of increase is closer to the factor 47 expected for collapse 



Figure 3. Mean accretion histories $\langle m(t)\rangle$ of groups of $\sim 10$ protostars having similar start times, in each of three turbulent fragmentation simulations (red curves). Black lines are linear fits to each $\langle m(t)\rangle$. The corresponding mass accretion rate is shown in units of the rate for a collapsing singular isothermal sphere (SIS). The rates greatly exceed the SIS rate (Myers et al. 2016). (A color version of this figure is available online.)

of an initially uniform isothermal sphere (Larson 1969, Penston 1969), but star-forming cores are observed to be centrally condensed. The constant rate also differs from the increasing rate expected for mass-dependent accretion models such as Bondi accretion (Bondi 1952).

The protostar mass accretion rates reported here resemble those in a numerical study of turbulent fragmentation, where shock formation of a massive filament drives supersonic flows into and along the filament (Banerjee et al. 2006).

\section{The end of turbulence}

It has been known for many years that some dense regions have spectral lines whose widths are trans-sonic or subsonic (Rydbeck et al. 1977; Myers 1983). In these regions, the large-scale supersonic motions that structure gas into filaments are relatively negligible. "Dense core" gas dominated by thermal motions is often considered an initial condition for isolated low-mass star formation (André et al. 2014).

Regions dominated by thermal motions were found to have typical size $0.1 \mathrm{pc}$ based on maps of $\mathrm{NH}_{3}$ line emission, a size similar to the thermal Jeans length for $10 \mathrm{~K}$ gas (Myers \& Benson 1983). The origin of such low-turbulence gas in large-scale regions of greater turbulence has been attributed to stagnation zones in colliding flows (BallesterosParedes et al. 1999; Mac Low \& Klessen 2004). Their 0.1-pc size scale regions is much greater than the mpc size scale for true dissipation of subsonic turbulence (Falgarone et al. 2009).

An important recent finding is that sonic motions are not confined only to 0.1-pc cores. Instead, sonic motions are now known to dominate $\mathrm{C}^{18} \mathrm{O}$ line widths in filamentary core environments in Taurus and in Musca. The filaments widths are again close to $0.1 \mathrm{pc}$, but their lengths can be many pc (Hacar \& Tafalla 2011; Hacar et al. 2013; Kainulainen et al. 2016; Fig. 4).

When such filaments have sufficient mass per length, they harbor dense cores of similar width and similar subsonic motions. The cores and their host filaments also share similar line center velocities, suggesting that they have together decoupled from the turbulent motions that formed them (Tafalla \& Hacar 2015). Matching these findings presents a challenge and an opportunity for turbulent simulations. 


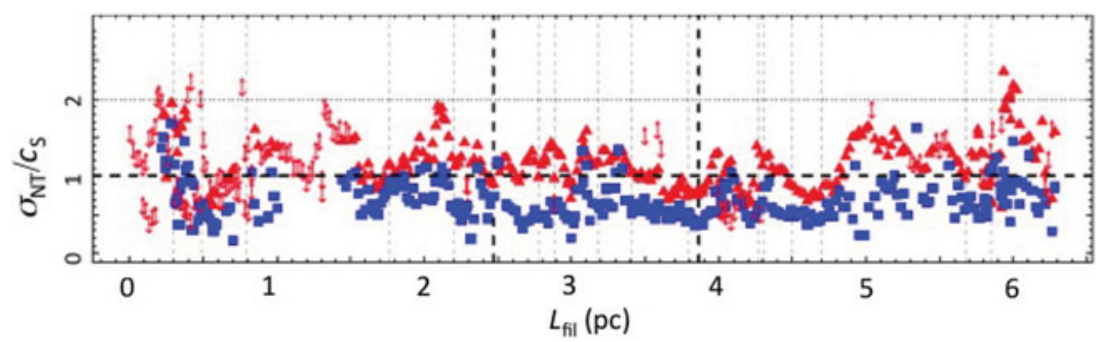

Figure 4. A sonic filament in Musca, extending over $\sim 6$ pc. The red and blue points indicate the ratio of opacity-corrected nonthermal velocity dispersion to sound speed, for lines of ${ }^{13} \mathrm{CO}$ and $\mathrm{C}^{18} \mathrm{O}$, respectively. Adapted from Kainulainen et al. (2016). (A color version of this figure is available online.)

\section{Conclusion}

The results discussed in Sections 2-5 suggest some possible answers to the questions posed in the introduction.

(1) Is cluster formation slow or fast? Cluster observations and simulations of turbulent fragmentation indicate that embedded clusters have a significant population of stars younger than $1 \mathrm{Myr}$. A quasi-equilibrium evolution has not been ruled out, but the majority of recent studies match embedded cluster properties with a "fast" dynamical picture involving supersonic flows.

(2) Do massive stars in clusters form first or last? Several turbulent simulations indicate that the most massive stars start their accretion early in the star-forming life of the cluster and have relatively long accretion durations. In this picture, massive stars are born early, but their powerful winds and radiation feedback do not arise until late in the cluster history, when they disperse dense gas and terminate further star formation.

(3) Do cluster protostars gain mass at a rate expected from models of "collapse" or "accretion"? In several simulations of hydrodynamic turbulence, the average $\langle m(t)\rangle$ of protostar accretion histories having similar start times shows protostar mass increasing at a nearly constant rate. This rate exceeds that of a collapsing singular isothermal sphere by one to two orders of magnitude, increasing with the level of initial supersonic turbulence. This rate does not increase with mass, in contrast with mass-dependent accretion models. Thus the typical gain rate is a constant, as in SIS collapse, but its magnitude is much greater than in SIS collapse models and is more typical of accretion models.

\section{Summary}

The main points of this review are:

Analytic models of cluster formation. Quasi-static contraction of a cluster-forming clump matches the star-formation rate in Orion as a function of time, over $\sim 10 \mathrm{Myr}$ (Tan et al. 2006; Huff \& Stahler 2007). The core mass function (CMF) observed in nearby starforming clouds is well matched by a model where cores are a subset of lognormal density fluctuations at all spatial scales, provided their density exceeds a turbulent Jeans criterion (Hennebelle \& Chabrier 2008; Hopkins 2012). The Kennicutt-Schmidt increase of star-formation rate surface density with gas surface density in galactic and extragalactic clouds is approximated by an extension of the CMF model (Chabrier et al. 2014).

Simulations of cluster formation by turbulent fragmentation. Evolution of a 1-pc clump having $500 M_{\odot}$, supersonic hydrodynamic turbulence, and hundreds of Jeans masses produces a filamentary network that makes more than 100 sink cells in an initial free-fall time, some still accreting and others that have stopped accreting. Their mass 
distribution matches the peak of the IMF and approximates its shape (Bate 2012, 2014). Another study addresses the low efficiency of star formation by evolving four initially identical clumps, until each has converted $20 \%$ of its mass into stars. The clumps collapse (1) with gravity alone, (2) with gravity and turbulence, (3) with gravity, turbulence, and magnetic fields, and (4) with gravity, turbulence, magnetic fields, and protostar outflow jets. The star-formation time increases by an order of magnitude from (1) to (4), bringing the resulting star-formation rate to within a factor of a few of the typical observed rate of a few $M_{\odot} \mathrm{pc}^{-2} \mathrm{Myr}^{-1}$ (Federrath 2015; see also Wang et al. 2010).

Accretion histories of cluster protostars. Comparison of three simulations of cluster formation by hydrodynamic turbulent fragmentation indicates that protostars are born at a typical rate of a few hundred per initial free-fall time. Those born early tend to have greater final masses, and their masses tend to increase linearly with time. Their mass accretion rate exceeds that due to a collapsing SIS by a factor 10-100, increasing with the initial turbulence of the simulation. Such a constant, superthermal accretion rate differs in magnitude from SIS collapse, and its constant magnitude differs from mass-dependent accretion models such as Bondi accretion, where the rate increases with mass and with time (Myers et al. 2015).

Extended regions of low turbulence. Recent observations indicate that dense gas with subsonic turbulence is not confined only to dense cores with size $\sim 0.1$ pc but also is seen in the filamentary environments of some cores, according to observations in lines of $\mathrm{C}^{18} \mathrm{O}$ (Hacar \& Tafalla 2011; Kainulainen et al. 2016). These filaments, in the nearby Taurus and Musca regions, extend up to several pc. The cores and filaments in these systems have similar spatial structures in their line centers and widths, suggesting that they have together decoupled from their turbulent environments. This result presents a challenge and opportunity to models of cloud turbulence.

\section{References}

Allen, L., Megeath, S., Gutermuth, R., Myers, P., Wolk, S., et al. 2007, in: B. Reipurth, D. Jewitt, \& K. Keil (eds.), Protostars and Planets $V$ (Tucson: U. of Arizona Press), p. 361

André, P., di Francesco, J., Ward-Thompson, D., Inutsuka, S., Pudritz, R., \& Pineda, J. 2014, in: H. Beuther, R. Klessen, C. Dullemond, \& T. Henning (eds.), Protostars and Planets VI, (Tucson: U. of Arizona Press), p. 27

Ballesteros-Paredes, J., Harmann, L., \& Vázquez-Semadeni, E. 1999, ApJ, 527, 285

Banerjee, R., Pudritz, R., \& Anderson, D. 2006, MNRAS, 373, 1091

Bate, M. 2012, MNRAS, 419, 3115

Bate, M. 2014, MNRAS, 442, 285

Bondi, H. 1952, MNRAS, 112, 195

Bonnell, I., Bate, M., Clarke, C., \& Pringle, J. 1997, MNRAS, 285, 201

Bonnell, I., Bate, M., Clarke, C., \& Pringle, J. 2001, MNRAS, 323, 785

Chabrier, G. 2005, in: E. Corbelli, F. Palla, \& H. Zinnecker (eds.), The Initial Mass Function 50 Years Later, Astrophysics and Space Science Library, v. 327 (Dordrecht: Springer), p. 41

Chabrier, G., Hennebelle, P., \& Charlot, S. 2014, ApJ, 796, 75

Elmegreen, B. 2000, ApJ, 530, 277

Falgarone, E., Pety, J., \& Hily-Blant, P. 2009, A\&A, 507, 355

Federrath, C. 2015, MNRAS, 450, 4035

Girichidis, P., Federrath, C., Banerjee, R., \& Klessen, R. 2011, MNRAS, 413, 2741

Hacar, A. \& Tafalla, M. 2011, A\&SA, 533, 34

Hacar, A., Tafalla, M., Kauffmann, J., \& Kovács, A. 2013, A\&3A, 554, A55

Hennebelle, P. \& Chabrier, G. 2008, ApJ, 684, 395

Herbig, G. 1962, ApJ, 135, 73

Hopkins, P. 2012, MNRAS, 423, 2037 
Huff, E. \& Stahler, S. 2007, ApJ, 666, 281

Kainulainen, J., Hacar, A., Alves, J., Beuther, H., Bouy, H., \& Tafalla, M. 2016, A\& A, 586, A27

Kennicutt, R. 1998, ApJ, 498, 541

Körtgen, B. \& Banerjee, R. 2015, MNRAS, 451, 334

Kroupa, P. 2001, MNRAS, 322, 231

Krumholz, M., Klein, R., \& McKee, C. 2012, ApJ, 754, 71

Krumholz, M., Bate, M., Arce, H., Dale, J., Gutermuth, R., Klein, R., Li, Z-Y., Nakamura, F., \& Zhang, Q. 2014, in: H. Beuther, R. Klessen, C. Dullemond, \& T. Henning (eds.), Protostars and Planets VI, (Tucson: U. of Arizona Press), p. 243

Larson, R. 1969, MNRAS, 145, 271

Longmore, S., Kruijssen, J., Bastian, N., Bally, J., Rathborne, J., Testi, L., Stolte, A., Dale, J., Bressert, E., \& Alves, J. 2014, in: H. Beuther, R. Klessen, C. Dullemond, \& T. Henning (eds.), Protostars and Planets VI, (Tucson: U. of Arizona Press), p. 291

Luhman, K. 2012, ARAA, 50, 65

Mac Low, M. \& Klessen, R. 2004, RvMP, 76, 125

McKee, C. \& Ostriker, E. 2007, ARAA, 45, 565

Myers, A., Klein, R., Krumholz, R., \& McKee, C. 2014, MNRAS, 439, 342

Myers, P. 1983, ApJ, 270, 105

Myers, P. \& Benson, P. 1983, ApJ, 266, 309

Myers, P., Bate, M., Elmegreen, B., Federrath, C., Girichidis, P., Klein, R., Klessen, R., Krumholz, M., Li, Z-Y., McKee, C., Offner, S., \& Ridge, N. 2016, in preparation

Offner, S., Clark, P., Hennebelle, P., Bastian, N., Bate, M., Hopkins, P., Moreaux, E., \& Whitworth, A. 2014, in: H. Beuther, R. Klessen, C. Dullemond, \& T. Henning (eds.), Protostars and Planets VI, (Tucson: U. of Arizona Press), p. 53

Palla, F. \& Stahler, S. 2000, ApJ, 540, 255

Penston, M. 1969, MNRAS, 144, 425

Portegies Zwart, S., McMillan, S., \& Gieles, M. 2010, ARAA, 48, 431

Rydbeck, O., Sume, A., Hjalmarson, A., Elldér, J., Rönnäng, B., \& Kollberg, E. 1977, ApJL, 215,35

Salpeter, E. 1955, ApJ, 121, 161

Schmidt, M. 1959, ApJ, 129, 243

Shu, F. 1977, ApJ, 214, 488

Shu, F., Adams, F., \& Lizano, S. 1987, ARAA, 25, 23

Tafalla, M. \& Hacar, A. 2015, A\&A, 574, 104

Tan, J., Krumholz, M., \& McKee, C. 2006, ApJ, 641, 121

Tan, J., Beltrán, M., Caselli, P., Fontani, F., Fuente, A., Krumholz, M., McKee, C., \& Stolte, A. 2014, in: H. Beuther, R. Klessen, C. Dullemond, \& T. Henning (eds.), Protostars and Planets VI, (Tucson: U. of Arizona Press), p. 149

Vázquez-Semadeni, E., Ryu, D., Passot, T., González, R., \& Gazol, A. 2006, ApJ, 643, 245

Wang, P., Li, Z-Y., Abel, T., \& Nakamura, F. 2010, ApJ, 709, 27

Zinnecker, H. 1982, Ann. NYAS, 395, 226

Zinnecker, H. \& Yorke, H. 2007, ARAA, 45, 481 